\title{
EDITORIAL
}

\section{Subspecialties of Cardiovascular Medicine}

Cardiovascular Medicine has evolved as a specialty during the second half of the 20th century. Several distinct subspecialties emerged - mainly as a result of scientiûc advances and a series of technological and procedural innovations relevant to patient care. Several different types of cardiologists provide speciûc services to patients and to other types of cardiologists. Fuster et al mentioned various types of cardiovascular specialists and their training requirements ${ }^{1}$. According to his article there are 10 types of cardiovascular specialists. These are mentioned below:

1. General Clinical Cardiologist

2. Interventional Cardiologist

3. Electrophysiologist

4. Echocardiologist

5. Nuclear Cardiologist

6. MR/CT Cardiologist

7. Heart Failure and Transplant Cardiologist

8. Preventive Cardiologist

9. Vascular Medicine Specialist

10. Cardiovascular Investigator

In USA each candidate has to undergo 3 year training with a board certified examination in internal medicine \& 3 year training followed by board certified examination in cardiovascular medicine or other certification examination. Those who want to be interventional cardiologist or electrophysiologist have to take additional 1 year training.

In Bangladesh in our MD Cardiology course we have 2 year course in internal medicine followed by an examination with another 3 year course in cardiology followed by another examination. Our course is designed to produce general clinical cardiologist with exposure to non-invasive and invasive procedures. At present we do not have provision for further training in subspecialties following MD in Cardiology. In our centre some cardiologists actually blend both clinical and interventional cardiology. Therefore the boundaries are not fixed. We do not have formally other subspecialties at present.

Many diseases in cardiovascular medicine require treatment by both clinical and interventional cardiologists. Some of the examples are mentioned below:

* Coronary artery disease: Both acute coronary syndrome $\&$ chronic ischaemic heart disease may require both medical and interventional management.

* Cardiac arrhythmias: Both bradyarrhythmias \& tachyarrhythmias may require antiarrhythmiac drugs \& interventional treatment in the form of cardiac pacing, electrophysiologic study and ablation, implantable cardioverter-defibrillator. Left atrial appendage occluder device may be inserted for prevention of thrombo-embolism in atrial fibrillation.

* Hypertension: Apart from medical management angioplasty with stenting may be required in renal artery stenosis and coarctation of aorta.

* Congenital heart disease: For the definite management of shunt anomalies various devices have been developed which is under the domain of interventional cardiologist.

* Heart Failure: In advanced heart failure there are indications of cardiac resynchronization therapy who is not responding to anti-failure medication.

* Cardiogenic shock: Apart from revascularization patient may require intra-aortic balloon pump and left ventricular assist device,

* Valvular heart disease: In mitral and pulmonary stenosis balloon valvuloplasty is indicated who meets certain criteria. There are also certain conditions where transcatheter aortic valve implantation is needed.

* Peripheral arterial disease: Patients suffering from peripheral arterial disease may need angioplasty in addition to medication.

From the above mentioned diseases we can see that patient suffering from various cardiovascular diseases need close cooperation and interaction between clinical and 
interventional cardiologists for the continuation of treatment. After many interventions patient needs to take a lot of medication which he or she used before intervention. Therefore many cardiologists practise both clinical and interventional cardiology although he may give most of the time to either sub-specialty depending upon the expertise.

In essence, the 'heart team' consists of both clinical and interventional cardiologists with input of various other cardiovascular sub-specialties for proper decision making process in the management of cardiovascular patients.

\section{Manzoor Mahmood}

Associate Professor, Department of Cardiology, BSMMU.

\section{Harisul Hoque}

Professor, Department of Cardiology, BSMMU.

\section{Reference:}

1. Valentin Fuster, MD, PHD, FACC, Chair, John W. Hirshfeld, Jr, MD, FACC, Co-Chair Alan S. Brown, MD, FACC, Bruce H. Brundage, MD, MACC, W. Bruce Fye, MD, MA, MACC, Richard P. Lewis, Working Group 8: Deûning the Different Types of Cardiovascular Specialists and Developing a New Model for Training General Clinical Cardiologists. JACC Vol. 44, No. 2, 2004:267-71 